\title{
Synthesis, characterization of biologically potent novel chalcones bearing urea, thiourea and acetamide linkages
}

\author{
Nirali S. Mewada, Dhruvin R. Shah, Kishor H. Chikhalia* \\ Department of Chemistry, School of Sciences, Gujarat University, \\ Ahmedabed-380009, Gujarat, India \\ *Tel: +91-79-26300969/9427155529; *Fax: +91-79-26308545 \\ *E-mail: chikhalia_kh@yahoo.com
}

\begin{abstract}
Three series of some novel chalcone based urea, thiourea and acetamide derivatives were designed, synthesized and screened for their antimicrobial and antifungal activities. All the synthesized compounds are first reported. The structures of the compounds were elucidated with the aid of elemental analysis and spectral methods including IR, ${ }^{1} \mathrm{H}-\mathrm{NMR}$ spectral data. The prepared compounds were evaluated for antibacterial activity against two Gram-positive bacteria (Staphylococcus aureus, Staphylococcus pyogenus), two Gram-negative bacteria (Pseudomonas aeruginosa, Escherichia coli). The title compounds were also investigated for their antifungal activity using the broth micro dilution method. The bioassay results showed that compounds a few compounds showed good to superior in vitro antibacterial and antifungal activity.
\end{abstract}

Keywords: Chalcone; urea; thiourea; acetamide; antimicrobial activity and antifungal activity

\section{INTRODUCTION}

A higher occurrence of opportunistic microbial infections caused by various bacteria due to the fruition and broaden of multidrug-resistant microorganisms has become a widespread medical problem. Such infections most frequently cause severe morbidity and transience in incapacitated and resistant compromised patients. Such infections nearly all frequently affect resistant compromised individuals, patients with malignancies, and transplant recipients. Moreover, with an signal of around two million deaths each year. These facts further emphasize the imperative necessity to find new effectual and secure compounds to maintain and improve the management and prevention of opportunistic microbial and tubercular infections in an innovative era of exhaustive infectious disease control, elimination, and eradication. Chalcones comprise an important class of natural products belonging to the flavonoid family, which exhibit motivating biological activities together with anti-inflammatory [1], anti bacterial [2], anti oxidant [3], anti malarial [4] and anti 
cancer [5]. Due to their abundance in plants and ease of synthesis, this class of compounds has generated great interest for possible therapeutic uses. They are also effective in vivo as cell proliferating inhibitors, antitumor promoting and chemo preventing agents [6]. The above literature survey led us to consider the chalcone nucleus as a possible scaffold

Chalcone based urea, thiourea and acetamide derivatives which are of substantial industrial importance, and are linked to a series of biological actions including herbicidal activity [7], inhibition of nitric oxide [8], antimicrobial [9], anti-HIV [10], anti-viral [11], HDL-elevating [12], and analgesic properties [13,14]. Some chalcone-urea derivatives exhibited anti-inflammatory [15] and anti-malarial activity [16].

In view of the need to discover potent novel antimicrobial agents, and our previous positive results on chalcones, we have synthesized novel chalcone thiourea, urea and acetamide derivatives and evaluated their antimicrobial and antifungal activity against different strains of bacteria and fungal using the broth micro dilution method and compared with some reference drugs.

\section{RESULTS AND DISCUSSION}

\section{1. Materials and Methods}

All reagents were of analytical grade and use directly. All the melting points were determined in open glass capillary and are uncorrected. Progress of reaction was monitored by thin layer chromatography (TLC) using silica gel-G coated aluminium plates $(0.5 \mathrm{~mm}$ thickness, Merck) and spots were visualized under UV radiation, purified by recrystalization and column chromatography. The IR spectra were recorded on BRUKER TENSOR Series using $\mathrm{KBr}$ pellets. ${ }^{1} \mathrm{H}$ NMR spectra were recorded on $300 \mathrm{MHz}$ BRUKER ULTRASHIELD using DMSO-d6 as a solvent and TMS as an internal reference and chemical shift values were expressed in $\delta$ ppm.

\section{2. Chemistry}<smiles>[R][R]1cccc(NC(=S)Nc2ccc(C(=O)/C=C/c3ccc4c(c3)OCO4)cc2)c1</smiles>

Scheme 1. Synthesis of the title compounds. (a) anhydrous DMF, KF, $\mathrm{CH}_{2} \mathrm{Cl}_{2}, 110-120{ }^{\circ} \mathrm{C}$ (b) 4-aminoactophenone, MSA, r.t (c) Ar-NCO, THF, reflux (d) Ar-NCS, THF, reflux (e) $\mathrm{Ar}-\mathrm{NHCOCH}_{2} \mathrm{Cl}$, THF, reflux. 
In this work, the synthesis of the series proceeded as depicted in Schemes 1. 1-[4-(3Benzo[1,3]dioxol-5-yl-2-propenone-1-yl)-phenyl]-3aryl urea (4a-4j), 1-[4-(3-Benzo[1,3] dioxol-5-yl-2-propenone-1-yl)-phenyl]-3aryl thiourea (5a-5j) and 2-[4-(3-Benzo[1,3]dioxol5-yl-2-propenone)-phenylamino]-N-aryl- acetamide (6a-6j) were prepared in three steps. The intermediate Benzo[1,3]dioxole-5-carbaldehyde 2 was prepared by known method from catechol 1 [17], which was condensed with 4-amino acetophenone in the presence MSA (Methyl Sulfonic Acid) at r.t to get the yield 1-(4-Amino-phenyl)-3-benzo[1,3]dioxol-5-ylpropenone 3 [18]. Compound $\mathbf{3}$ was condensed with various isocyanate isothiocyanate and 2chloro-N-aryl acetamide to afforded $(\mathbf{4 a - 4 j}),(\mathbf{5 a - 5 j})$ and $(\mathbf{6 a - 6 j})$ respectively. All the synthesized compounds were fully characterized by IR, ${ }^{1} \mathrm{H}-\mathrm{NMR}$ spectroscopy and elemental analysis [19-25].

\section{EXPERIMENTAL SECTION}

\section{1. Preparation of Benzo[1,3]dioxole-5-carbaldehyde (2)}

A solution of catechol $1(1.0 \mathrm{~g}, 0.0090 \mathrm{~mol})$ in $300 \mathrm{ml}$ anhydrous DMF $(30 \mathrm{~mL})$ was shaken with of KF $(0.72 \mathrm{~g}, 0.0045 \mathrm{~mol})$ of and the mixture warmed up somewhat. $\mathrm{CH}_{2} \mathrm{Cl}_{2}$ $(0.85 \mathrm{~g}, 0.0099 \mathrm{~mol})$ was then added to the cooled solution, and the mixture heated at $110-$ $120{ }^{\circ} \mathrm{C}$ with an efficient reflux condenser for 2-3 hours. The cooled reaction mixture was extracted with ether, and the etheral extracts washed with water to remove DMF and with cold $5 \% \mathrm{NaOH}$. The solution was dried over $\mathrm{MgSO}_{4}$, and the solvent removed by evaporation. The residue was extracted with hot hexane followed by cooling, and evaporation of the extracts to give a $90 \%$ yield of 1,3-benzodioxole. M.P.: $37^{\circ} \mathrm{C}$.

\section{2. Preparation of 1-(4-Amino-phenyl)-3-benzo[1,3]dioxol-5-yl-propenone (3)}

4-amino acetophenone (5 g, $0.0369 \mathrm{~mol}$ ) as allowed to stir with Benzo[1,3]dioxole-5carbaldehyde $(4.21,0.0281 \mathrm{~mol})$ in MSA $(7 \mathrm{ml}, 0.1476)$ for $5 \mathrm{~min}$ at room temperature. The reaction mixture was diluted with water. The organic layer was washed with brine and dried over anhydrous sodium sulfate. The progress of reaction was monitored by TLC using toluene:ethyl acetate (6:4) as eluent. Solvent was removed under vacuum and crude product was purified by column chromatography to yield $60 \%$. M.P.: $145^{\circ} \mathrm{C}$.

\section{3. General Preparation of 1-[4-(3-Benzo[1,3]dioxol-5-yl-2-propenone-1-yl)-phenyl]- 3aryl urea $(\mathbf{4 a}-\mathbf{4} \mathbf{j})$}

A mixture of $3(2.1 \mathrm{~g}, 0.00783 \mathrm{~mol})$ and aryl isocyanate $(00783 \mathrm{~mol})$ in THF $(10 \mathrm{~mL})$ was refluxed for 5-6 hours. The progress of reaction was monitored by TLC using toluene: acetone $(7: 3)$ as eluent. After the completion of reaction, the solvent was removed by distillation and the resulting solid was recrystallized from methanol.

\section{4. Spectral data}

[1-[4-(3-Benzo[1,3]dioxol-5-yl-2-propenone-1-yl)-phenyl]-3-phenyl-urea (4a)

${ }^{1}$ H NMR: 4.78(s, 2H), 5.74(d, 1H), 5.66(d, 1H), 6.10(d, 1H), 6.06(d, 1H), 6.42(m, 1H), $6.49(\mathrm{~d}, 2 \mathrm{H}), 6.74(\mathrm{~d}, 2 \mathrm{H}), 7.00(\mathrm{dd}, 2 \mathrm{H}), 7.08(\mathrm{ddd}, 2 \mathrm{H}), 7.77(\mathrm{ddd}, 1 \mathrm{H}), 7.89(\mathrm{~s}, 1 \mathrm{H}), 7.96(\mathrm{~s}$, 1H). IR (KBr cm $\left.\mathbf{~ c m}^{-1}\right)$ : 1680(-C=O), 1490(-C=C), 1214(-C-O), 2969(-C-H), 3060(-C-H), 1605(-NH), 3462(-CO-NH). Anal. Calcd. For $\mathrm{C}_{23} \mathrm{H}_{18} \mathrm{~N}_{2} \mathrm{O}_{4}: \mathrm{C}, 71.49 ; \mathrm{H}, 4.70 ; \mathrm{N}, 7.25$, Found: $\mathrm{C}, 71.44 ; \mathrm{H}, 4.65 ; \mathrm{N}, 7.20, \mathbf{m p ~} 173{ }^{\circ} \mathrm{C}$, Yield: $83 \%$ 
1-[4-(3-Benzo[1,3]dioxol-5-yl-2-propenone-1-yl)-phenyl]-3-(2-fluoro-phenyl)-urea (4b)

${ }^{1}$ H NMR: $\left(400 \mathrm{MHz}, \mathrm{DMSO}-d_{6}\right) \delta$ ppm: 3.64(s, 2H), 4.69(d, 1H), 4.81(d, 1H), 7.3(d, 1H), 7.34(d, 1H), 7.44(s, 1H), 7.46(d, 2H), 7.51(d, 2H),7.79(dd, 1H), 8.0(dd, 1H), 8.18(dd, 1H), 8.21(dd, 1H), 9.16(s, 1H) \& 9.49(s, 1H). IR $\left(\mathbf{K B r ~ c m}^{-1}\right): 1680(-\mathrm{C}=\mathrm{O}), 1493(-\mathrm{C}=\mathrm{C}), 1210(-$ C-O), 2968(-C-H), 3060(-C-H), 1602(-NH), 3465(-CO-NH), 3060 (C-H), 782(-C-F) Anal. Calcd. For $\mathrm{C}_{23} \mathrm{H}_{17} \mathrm{FN}_{2} \mathrm{O}_{4}$ : C, 68.25; H, 4.20; N, 6.92 Found: C, 68.25; H, 4.15; N, 6.87. mp $171{ }^{\circ} \mathrm{C}$, Yield: $85 \%$

1-[4-(3-Benzo[1,3]dioxol-5-yl-2-propenone-1-yl)-phenyl]-3-(3-fluoro-phenyl)-urea (4c) ${ }^{1}$ H NMR: $\left(400 \mathrm{MHz}, \mathrm{DMSO}-d_{6}\right) \delta \mathrm{ppm}: 3.68(\mathrm{~s}, 2 \mathrm{H}), 4.74(\mathrm{~d}, 1 \mathrm{H}), 4.81(\mathrm{~d}, 1 \mathrm{H}), 7.41(\mathrm{~d}, 1 \mathrm{H})$, 7.45(s, 1H), 7.6(d, 1H), 7.52(d, 2H), 7.68(s, 2H), 7.72(s, 1H), 7.78(dd, 1H), 8.03(dd, 1H), 8.14(ddd, 1H), 9.21(s, 1H) \& 9.54(s, 1H). IR $\left(\mathbf{K B r} \mathbf{~ c m}^{-1}\right): 1682(-\mathrm{C}=\mathrm{O}), 1495(-\mathrm{C}=\mathrm{C}), 1212$ (C-O), 2960(-C-H), 3064(C-H), 1606(-NH), 3468(-CO-NH), 3062(C-H), 820(C-F). Anal. Calcd. For $\mathrm{C}_{23} \mathrm{H}_{17} \mathrm{FN}_{2} \mathrm{O}_{4}$ : C, 68.25; H, 4.20; N, 6.92 Found: C, 68.25; H, 4.15; N, 6.87. mp $173{ }^{\circ} \mathrm{C}$, Yield: $74 \%$

1-[4-(3-Benzo[1,3]dioxol-5-yl-2-propenone-1-yl)-phenyl]-3-(4-fluoro-phenyl)-urea(4d)

${ }^{1}$ H NMR: 4.83(s, 2H), 6.76(d, 1H), 6.77(d, 1H), 7.23(d, 1H), 7.23(d, 1H), 7.43(d, 1H), 7.44(d, 2H), 7.62(d, 2H), 7.83(d, 2H), 8.25(d, 2H), 9.0(s, 1H), 9.4(s, 1H). IR $\left(\mathbf{K B r} \mathbf{~ c m}^{-1}\right)$ : 1682(-C=O), 1495(-C=C), $1212(-\mathrm{C}-\mathrm{O}), 2960(-\mathrm{C}-\mathrm{H}), 3064(\mathrm{C}-\mathrm{H}), 1606(-\mathrm{NH}), 3468(-\mathrm{CO}-\mathrm{NH})$, 3062(C-H), 912(C-F). Anal. Calcd. For $\mathrm{C}_{23} \mathrm{H}_{17} \mathrm{FN}_{2} \mathrm{O}_{4}$ : C, 68.25; H, 4.20; N, 6.92 Found: C, 68.25; H, 4.15; N, 6.87. mp $180{ }^{\circ} \mathrm{C}$, Yield: $68 \%$

1-[4-(3-Benzo[1,3] dioxol-5-yl-2-propenone-1-yl)-phenyl]-3-o-tolyl-urea (4e)

${ }^{1} \mathbf{H}$ NMR : 2.32(s, $\left.1 \mathrm{H}\right), 3.59(\mathrm{~s}, 2 \mathrm{H}), 4.76(\mathrm{~d}, 1 \mathrm{H}), 4.81(\mathrm{~d}, 1 \mathrm{H}), 7.43(\mathrm{~d}, 1 \mathrm{H}), 7.48(\mathrm{~s}, 1 \mathrm{H})$, $7.58(\mathrm{~s}, 1 \mathrm{H}), 7.49(\mathrm{~d}, 2 \mathrm{H}), 7.59(\mathrm{~s}, 2 \mathrm{H}), 7.69(\mathrm{dd}, 1 \mathrm{H}), 7.72(\mathrm{ddd}, 1 \mathrm{H}), 7.74(\mathrm{dd}, 1 \mathrm{H}), 7.79(\mathrm{ddd}$, $1 \mathrm{H}), 8.68(\mathrm{~s}, 1 \mathrm{H}), 9.06(\mathrm{~s}, 1 \mathrm{H})$. IR $\left(\mathbf{K B r} \mathbf{~ m}^{-1}\right):$ 1685(-C=O), 1485(-C=C), 1209(-C-O), 2959(-C-H), 3044(C-H), 1601(-NH), 3470(-CO-NH), 3067(C-H), 580(Ar- $\left.\mathrm{CH}_{3}\right)$. Anal. Calcd. For $\mathrm{C}_{24} \mathrm{H}_{20} \mathrm{~N}_{2} \mathrm{O}_{4}$ : C, 71.97; H, 4.99; N, 6.99 Found: C, 71.92; H, 4.94; N, 6.94. mp $161{ }^{\circ} \mathrm{C}$, Yield: $62 \%$

1-[4-(3-Benzo[1,3]dioxol-5-yl-2-propenone-1-yl)-phenyl]-3-m-tolyl-urea (4f)

${ }^{1}$ H NMR: $2.36(\mathrm{~s}, 3 \mathrm{H}), 3.62(\mathrm{~s}, 2 \mathrm{H}), 4.76(\mathrm{~d}, 1 \mathrm{H}), 4.79(\mathrm{~d}, 1 \mathrm{H}), 7.4(\mathrm{~d}, 1 \mathrm{H}), 7.41(\mathrm{~s}, 1 \mathrm{H}), 7.54(\mathrm{~s}$, $1 \mathrm{H}), 7.47(\mathrm{~d}, 2 \mathrm{H}), 7.6(\mathrm{~s}, 2 \mathrm{H}), 7.6(\mathrm{~s}, 1 \mathrm{H}), 7.68(\mathrm{dd}, 1 \mathrm{H}), 7.71(\mathrm{dd}, 1 \mathrm{H}), 7.81(\mathrm{dd}, 1 \mathrm{H}), 8.78(\mathrm{~s}$, $1 \mathrm{H}), 9.13(\mathrm{~s}, 1 \mathrm{H}) . \mathbf{I R}\left(\mathbf{K B r} \mathbf{~ c m}^{-1}\right): 1662(-\mathrm{C}=\mathrm{O}), 1492(-\mathrm{C}=\mathrm{C}), 1210(-\mathrm{C}-\mathrm{O}), 2969(-\mathrm{C}-\mathrm{H})$, 3061(C-H), 1606(-NH), $3460(-\mathrm{CO}-\mathrm{NH}), 3065(\mathrm{C}-\mathrm{H}), 512\left(\mathrm{Ar}-\mathrm{CH}_{3}\right)$ Anal. Calcd. For $\mathrm{C}_{24} \mathrm{H}_{20} \mathrm{~N}_{2} \mathrm{O}_{4}$ : C, 71.97; H, 4.99; N, 6.99 Found: C, 71.92; H, 4.94; N, 6.94. mp $159{ }^{\circ} \mathrm{C}$, Yield: $71 \%$

1-[4-(3-Benzo[1,3]dioxol-5-yl-2-propenone-1-yl)-phenyl]-3-p-tolyl-urea (4g)

${ }^{1}$ H NMR: $2.36(\mathrm{~s}, 3 \mathrm{H}), 3.58(\mathrm{~s}, 2 \mathrm{H}), 4.23(\mathrm{~d}, 1 \mathrm{H}), 4.81(\mathrm{~d}, 1 \mathrm{H}), 7.24(\mathrm{~d}, 1 \mathrm{H}), 7.38(\mathrm{~s}, 1 \mathrm{H}), 7.51(\mathrm{~s}$, 1H), 7.44(d, 2H), 7.59(s, 2H), 7.54(d, 2H), 7.69(d, 2H), 8.9(s, 1H), 9.24(s, 1H). IR (KBr cm ${ }^{-}$ 1): 1692(-C=O), 1490(-C=C), 1214(-C-O), 2966(-C-H), 3069(C-H), 1606(-NH), 3462(-CO$\mathrm{NH}), 3067(\mathrm{C}-\mathrm{H}), 612\left(\mathrm{Ar}-\mathrm{CH}_{3}\right)$. Anal.Calcd. For $\mathrm{C}_{24} \mathrm{H}_{20} \mathrm{~N}_{2} \mathrm{O}_{4}: \mathrm{C}, 71.97 ; \mathrm{H}, 4.99 ; \mathrm{N}, 6.99$ Found: C, 71.92; H, 4.94; N, 6.94. mp $166{ }^{\circ} \mathrm{C}$, Yield: $65 \%$ 
1-[4-(3-Benzo[1,3]dioxol-5-yl-2-propenone-1-yl)-'phenyl]-3-(2-chloro-phenyl)-urea (4h) ${ }^{1}$ H NMR: 3.61(s, 2H), 4.71(d, 1H), 4.79(d, 1H), 7.28(d, 1H), 7.34(d, 1H), 7.41(s, 1H), 7.46(d, 2H), 7.52(d, 2H), 7.8(dd, 1H), 7.92(dd, 1H), 8.09(dd, 1H), 8.18(dd, 1H), 9.2( s, 1H), 9.51(s, 1H). IR (KBr cm $\left.{ }^{-1}\right): 1695(-\mathrm{C}=\mathrm{O}), 1497(-\mathrm{C}=\mathrm{C}), 1211(-\mathrm{C}-\mathrm{O}), 2962(-\mathrm{C}-\mathrm{H}), 3069(\mathrm{C}-$ $\mathrm{H}), 1608(-\mathrm{NH}), 3476(-\mathrm{CO}-\mathrm{NH}), 3064(\mathrm{C}-\mathrm{H}), 726(\mathrm{C}-\mathrm{Cl})$. Anal. Calcd. For $\mathrm{C}_{23} \mathrm{H}_{17} \mathrm{ClN}_{2} \mathrm{O}_{4}$ : C, 65.58; H, 4.03; N, 6.65 Found: C, 65.53; H, 3.98; N, 6.60. mp $173{ }^{\circ} \mathrm{C}$, Yield: $81 \%$

1-[4-(3-Benzo[1,3]dioxol-5-yl-2-propenone-1-yl)-'phenyl]-3-(3-chloro-phenyl)-urea (4i) ${ }^{1}$ H NMR: $3.66(\mathrm{~s}, 2 \mathrm{H}), 4.72(\mathrm{~d}, 1 \mathrm{H}), 4.81(\mathrm{~d}, 1 \mathrm{H}), 7.19(\mathrm{~d}, 1 \mathrm{H}), 7.29(\mathrm{~d}, 1 \mathrm{H}), 7.44(\mathrm{~s}, 1 \mathrm{H})$, 7.49(d, 2H), 7.54(d, 2H), 7.68(s, 1H), 7.79(dd, 1H), 8.09(dd, 1H), 8.14(ddd, 1H), 9.21(s, 1H), 9.64(s, 1H). IR (KBr cm $\left.{ }^{-1}\right)$ : 1680(-C=O), 1490(-C=C), 1214(-C-O), 3106(-C-H), 2955(C$\mathrm{H}), 1605(-\mathrm{NH}), 3446(-\mathrm{CO}-\mathrm{NH}), 3062(\mathrm{C}-\mathrm{H}), 780(\mathrm{C}-\mathrm{Cl})$. Anal. Calcd. For $\mathrm{C}_{23} \mathrm{H}_{17} \mathrm{ClN}_{2} \mathrm{O}_{4}$ : C, 65.58; H, 4.03; N, 6.65 Found: C, 65.53; H, 3.98; N, 6.60. mp $175{ }^{\circ} \mathrm{C}$, Yield: $79 \%$

1-[4-(3-Benzo[1,3]dioxol-5-yl-2-propenone-1-yl)-'phenyl]-3-(4-chloro-phenyl)-urea (4j) ${ }^{1}$ H NMR: 3.7(s, 2H) , 4.74(d, 1H), 4.84(d, 1H), 7.21(d, 1H), 7.26(d, 1H), 7.49(s, 1H), 7.51(d, $2 \mathrm{H}), 7.59(\mathrm{~d}, 2 \mathrm{H}), 7.89(\mathrm{~d}, 2 \mathrm{H}), 8.32(\mathrm{~d}, 2 \mathrm{H}), 9.13(\mathrm{~s}, 1 \mathrm{H}), 9.62(\mathrm{~s}, 1 \mathrm{H})$. IR $\left(\mathbf{K B r ~ c m}^{-1}\right)$ : $1665(-$ $\mathrm{C}=\mathrm{O}), \quad 1487(-\mathrm{C}=\mathrm{C}), \quad 1215(-\mathrm{C}-\mathrm{O}), \quad 2960(-\mathrm{C}-\mathrm{H}), \quad 3069(\mathrm{C}-\mathrm{H}), \quad 1608(-\mathrm{NH}), \quad 3462(-\mathrm{CO}-\mathrm{NH})$, $3060(\mathrm{C}-\mathrm{H}), 825(\mathrm{C}-\mathrm{Cl})$. Anal. Calcd. For $\mathrm{C}_{23} \mathrm{H}_{17} \mathrm{ClN}_{2} \mathrm{O}_{4}: \mathrm{C}, 65.58 ; \mathrm{H}, 4.03$; N, 6.65 Found: $\mathrm{C}, 65.53 ; \mathrm{H}, 3.98 ; \mathrm{N}, 6.60 . \mathbf{m p} 178^{\circ} \mathrm{C}$, Yield: $85 \%$

\section{5. General Preparation of 1-[4-(3-Benzo[1,3]dioxol-5-yl-2-propenone-1-yl)-phenyl]- 3aryl thiourea (5a-5j)}

A mixture of $3(2.1 \mathrm{~g}, 0.00783 \mathrm{~mol})$ and aryl isothiocyanate $(00783 \mathrm{~mol})$ in THF (10 $\mathrm{mL}$ ) was refluxed for 5-6 hours. The progress of reaction was monitored by TLC using toluene: acetone (6:4) as eluent. After the completion of reaction, the solvent was removed by distillation and the resulting solid was recrystallized from methanol.

1-[4-(3-Benzo[1,3]dioxol-5-yl-2-propenone-1-yl)-phenyl]-3-phenyl-thiourea (5a)

${ }^{1}$ H NMR: $4.81(\mathrm{~s}, 2 \mathrm{H}), 5.71(\mathrm{~d}, 1 \mathrm{H}), 5.73(\mathrm{~d}, 1 \mathrm{H}), 6.13(\mathrm{~d}, 1 \mathrm{H}), 6.16(\mathrm{~d}, 1 \mathrm{H}), 6.38(\mathrm{~m}, 1 \mathrm{H})$, 6.43(d, 2H), 6.69(d, 2H), 6.97(dd, 2H), 7.04(ddd, 2H), 7.72(ddd, 1H), 7.87(s, 1H), 7.91( s, 1H). IR $\left(\mathbf{K B r ~ c m}^{-1}\right):$ 1650(-C=O), 1490(-C=C), 1214(-C-O), 1530(C=S), 3410(-NH), 3276 (-CS-NH), 2969 (-C-H), $3060(-\mathrm{C}-\mathrm{H})$. Anal.Calcd. For $\mathrm{C}_{23} \mathrm{H}_{18} \mathrm{~N}_{2} \mathrm{O}_{3} \mathrm{~S}: \mathrm{C}, 68.57 ; \mathrm{H}, 4.47$; N, 6.95 Found: C, 68.61; H, 4.51; N, 6.90. mp $190{ }^{\circ} \mathrm{C}$, Yield: $78 \%$

\section{1-[4-(3-Benzo[1,3]dioxol-5-yl-2-propenone-1-yl)-phenyl]-3-(2-fluoro-phenyl)-thiourea} (5b)

${ }^{1}$ H NMR: 4.86(s, 2H), 5.64(d, 1H), 5.72(d, 1H), 6.14(d, 1H), 6.18(d, 1H), 6.24(s, 1H), 6.43(d, 2H), 6.59(d, 2H), 6.98(dd, 1H), 7.05(dd, 1H), 7.13(d, 1H), 7.17(dd, 1H), 7.83(s, 1H), 7.89( s, 1H). IR (KBr cm $\left.{ }^{-1}\right)$ : $1685(-\mathrm{C}=\mathrm{O}), 1504(-\mathrm{C}=\mathrm{C}), 1223(-\mathrm{C}-\mathrm{O}), 1538(\mathrm{C}=\mathrm{S}), 3412(-$ $\mathrm{NH}), 3272(-\mathrm{CS}-\mathrm{NH}), 2971(-\mathrm{C}-\mathrm{H}), 3063(-\mathrm{C}-\mathrm{H}), 814(\mathrm{C}-\mathrm{F})$. Anal. Calcd. For $\mathrm{C}_{23} \mathrm{H}_{17} \mathrm{FN}_{2} \mathrm{O}_{3} \mathrm{~S}$ : C, 65.64; H, 4.04; N, 6.65 Found: C, 65.69; H, 4.09; N, 6.60. mp $182{ }^{\circ} \mathrm{C}$, Yield: $78 \%$

1-[4-(3-Benzo[1,3]dioxol-5-yl-2-propenone-1-yl)-phenyl]-3-(3-fluoro-phenyl)- thiourea (5c)

${ }^{1}$ H NMR: 4.89(s, 2H), 5.65(d, 1H), 5.71(d, 1H), 6.12(d, 1H), 6.17(d, 1H), 6.25(s, 1H), 6.45(d, 2H), 6.62(d, 2H), 6.95(s, 1H), 6.98(dd, 1H), 7.04(dd, 1H), 7.14(dd, 1H), 7.89(s, 1H),

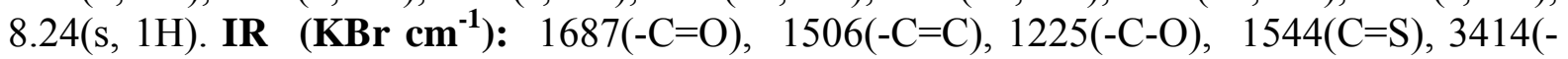


$\mathrm{NH}), 3275(-\mathrm{CS}-\mathrm{NH}), 2974(-\mathrm{C}-\mathrm{H}), 3065(-\mathrm{C}-\mathrm{H}), 729(\mathrm{C}-\mathrm{F})$. Anal. Calcd. For $\mathrm{C}_{23} \mathrm{H}_{17} \mathrm{FN}_{2} \mathrm{O}_{3} \mathrm{~S}$ : C, 65.64; H, 4.04; N, 6.65 Found: C, 65.59; H, 4.00; N, 6.70. mp $176{ }^{\circ} \mathrm{C}$, Yield: $80 \%$

1-[4-(3-Benzo[1,3]dioxol-5-yl-2-propenone-1-yl)-phenyl]-3-(4-fluoro-phenyl)- thiourea (5d)

${ }^{1}$ H NMR: $4.91(\mathrm{~s}, 2 \mathrm{H}), 5.63(\mathrm{~d}, 1 \mathrm{H}), 5.69(\mathrm{~d}, 1 \mathrm{H}), 6.11(\mathrm{~d}, 1 \mathrm{H}), 6.18(\mathrm{~d}, 1 \mathrm{H}), 6.24(\mathrm{~s}, 1 \mathrm{H})$, 6.47(d, 2H), 6.64(d, 2H), 6.96(d, 2H), 7.08(d, 2H), 7.84( s, 1H), 8.03( s, 1H). IR $\left(\mathbf{K B r ~ c m}^{-1}\right)$ : 1672(-C=O), 1509(-C=C), 1228(-C-O), 1548(C=S), 3416(-NH), 3277(-CS-NH), 2978(-C-H), 3067(-C-H), 830(C-F). Anal. Calcd. For $\mathrm{C}_{23} \mathrm{H}_{17} \mathrm{FN}_{2} \mathrm{O}_{3} \mathrm{~S}$ : C, 65.64; H, 4.04; N, 6.65 Found: $\mathrm{C}, 65.71 ; \mathrm{H}, 4.08 ; \mathrm{N}, 6.58 . \mathbf{m p} 177^{\circ} \mathrm{C}$, Yield: $75 \%$

\section{1-[4-(3-Benzo[1,3]dioxol-5-yl-2-propenone-1-yl)-phenyl]-3-o-tolyl-thiourea (5e)}

${ }^{1}$ H NMR: 2.34(s, 3H), 4.88(s, 2H), 5.61(d, 1H), 5.7(d, 1H), 6.09(d, 1H), 6.14(d, 1H), 6.29(m, $1 \mathrm{H}), 6.46(\mathrm{~d}, 2 \mathrm{H}), 6.67(\mathrm{~d}, 2 \mathrm{H}), 6.94(\mathrm{~d}, 1 \mathrm{H}), 6.96(\mathrm{dd}, 1 \mathrm{H}), 6.99(\mathrm{dd}, 1 \mathrm{H}), 7.03(\mathrm{dd}, 1 \mathrm{H}), 7.84(\mathrm{~s}$, $1 \mathrm{H}), 8.01(\mathrm{~s}, 1 \mathrm{H})$. IR $\left(\mathbf{K B r} \mathbf{~ c m}^{-1}\right): 1675(-\mathrm{C}=\mathrm{O}), 1512(-\mathrm{C}=\mathrm{C}), 1231(-\mathrm{C}-\mathrm{O}), 1551(\mathrm{C}=\mathrm{S}), 3418(-$ $\mathrm{NH}), \quad 3279(-\mathrm{CS}-\mathrm{NH}), \quad 2983(-\mathrm{C}-\mathrm{H}), \quad 3071(-\mathrm{C}-\mathrm{H}), \quad 519\left(\mathrm{Ar}_{-}-\mathrm{CH}_{3}\right)$. Anal. Calcd. For $\mathrm{C}_{24} \mathrm{H}_{20} \mathrm{~N}_{2} \mathrm{O}_{3} \mathrm{~S}$ : C, 69.14; H, 4.80; N, 6.72 Found: C, 69.20; H, 4.85; N, 6.78. mp $183{ }^{\circ} \mathrm{C}$, Yield: $77 \%$

1-[4-(3-Benzo[1,3]dioxol-5-yl-2-propenone-1-yl)-phenyl]-3-m-tolyl-thiourea (5f)

${ }^{1}$ H NMR: 2.38(s, 3H), 4.89(s, 2H), 5.63(d, 1H), 5.71(d, 1H), 5.96(d, 1H), 6.04(d, 1H), 6.13(m, 1H), 6.43(d, 2H), 6.68(d, 2H), 6.93(m, 1H), 6.97(d, 1H), 7.02(d, 1H), 7.1(dd, 1H), 7.84(s, 1H), 8.21(s, 1H). IR $\left(\mathbf{K B r ~ c m}^{-1}\right): 1678(-\mathrm{C}=\mathrm{O}), 1515(-\mathrm{C}=\mathrm{C}), 1233(-\mathrm{C}-\mathrm{O}), 1554(\mathrm{C}=\mathrm{S})$, 3421(-NH), 3232(-CS-NH), 2985(-C-H), 3075(-C-H), 585(Ar-CH $\mathrm{CH}_{3}$. Anal. Calcd. For $\mathrm{C}_{24} \mathrm{H}_{20} \mathrm{~N}_{2} \mathrm{O}_{3} \mathrm{~S}$ : C, 69.14; H, 4.80; N, 6.72 Found: C, 69.10; H, 4.75; N, 6.65. mp $188{ }^{\circ} \mathrm{C}$, Yield: $69 \%$

1-[4-(3-Benzo[1,3]dioxol-5-yl-2-propenone-1-yl)-phenyl]-3-p-tolyl-thiourea $(5 \mathrm{~g})$

${ }^{1}$ H NMR: $2.34(\mathrm{~s}, 3 \mathrm{H}), 4.79(\mathrm{~s}, 2 \mathrm{H}), 4.98(\mathrm{~d}, 1 \mathrm{H}), 5.78(\mathrm{~d}, 1 \mathrm{H}), 5.89(\mathrm{~d}, 1 \mathrm{H}), 6.31(\mathrm{~m}, 1 \mathrm{H})$, $6.47(\mathrm{~d}, 2 \mathrm{H}), 6.58(\mathrm{~d}, 2 \mathrm{H}), 6.84(\mathrm{~d}, 2 \mathrm{H}), 6.91(\mathrm{~d}, 2 \mathrm{H}), 7.8(\mathrm{~s}, 1 \mathrm{H}), 8.21(\mathrm{~s}, 1 \mathrm{H}) . \mathbf{I R}\left(\mathbf{K B r} \mathbf{~ c m}^{-1}\right)$ : 1681 $(-\mathrm{C}=\mathrm{O}), 1517(-\mathrm{C}=\mathrm{C}), 1235(-\mathrm{C}-\mathrm{O}), 1555(\mathrm{C}=\mathrm{S}), 3423(-\mathrm{NH}), 3235(-\mathrm{CS}-\mathrm{NH}), 2987(-\mathrm{C}-\mathrm{H})$, 3078(-C-H), 676(Ar-CH 3$)$. Anal. Calcd. For $\mathrm{C}_{24} \mathrm{H}_{20} \mathrm{~N}_{2} \mathrm{O}_{3} \mathrm{~S}$ : C, 69.14; H, 4.80; N, 6.72 Found: C, 69.22; H, 4.86; N, 6.78. mp $191{ }^{\circ} \mathrm{C}$, Yield: $72 \%$

1-[4-(3-Benzo[1,3]dioxol-5-yl-2-propenone-1-yl)-'phenyl]-3-(2-chloro-phenyl)- thiourea (5h)

${ }^{1}$ H NMR: 4.94(s, 2H), 5.66(d, 1H), 5.71(d, 1H), 6.13(d, 1H), 6.18(d, 1H), 6.29(m, 1H), 6.42(d, 2H), 6.62(d, 2H), 6.96(dd, 1H), 6.99(dd, 1H), $7.06(\mathrm{dd}, 1 \mathrm{H}), 7.14(\mathrm{dd}, 1 \mathrm{H}), 7.85(\mathrm{~s}$, $1 \mathrm{H}), 7.89(\mathrm{~s}, 1 \mathrm{H})$. IR $\left(\mathbf{K B r ~ c m}^{-1}\right)$ : 1678(-C=O), 1493(-C=C), 1210(-C-O), 1532(C=S), 3429($\mathrm{NH}), \quad 3275(-\mathrm{CS}-\mathrm{NH}), \quad 2960(-\mathrm{C}-\mathrm{H}), \quad 3062(-\mathrm{C}-\mathrm{H}), \quad 726(\mathrm{C}-\mathrm{Cl}) . \quad$ Anal. Calcd. For $\mathrm{C}_{23} \mathrm{H}_{17} \mathrm{ClN}_{2} \mathrm{O}_{3} \mathrm{~S}$ : C, 63.17; H, 3.89; N, 6.40 Found: C, 63.12; H, 3.92; N, 6.45. mp $187{ }^{\circ} \mathrm{C}$, Yield: $65 \%$

1-[4-(3-Benzo[1,3]dioxol-5-yl-2-propenone-1-yl)-'phenyl]-3-(3-chloro-phenyl)- thiourea (5i)

${ }^{1}$ H NMR: 4.98(s, 2H), 5.65(d, 1H), 5.69(d, 1H), 6.19(d, 1H), 6.21(d, 1H), 6.31(s, 1H), 6.44(d, 2H), 6.71(d, 2H), 6.91(m, 1H), 6.94(dd, 1H), 6.96(dd, 1H), 7.04(ddd, 1H), 7.86(s, $1 \mathrm{H}), 8.15(\mathrm{~s}, 1 \mathrm{H})$. IR $\left(\mathbf{K B r} \mathbf{~ c m}^{-1}\right): 1685(-\mathrm{C}=\mathrm{O}), 1507(-\mathrm{C}=\mathrm{C}), 1212(-\mathrm{C}-\mathrm{O}), 1530(\mathrm{C}=\mathrm{S}), 3400(-$ 
$\mathrm{NH}), \quad 3278(-\mathrm{CS}-\mathrm{NH}), \quad 2979(-\mathrm{C}-\mathrm{H}), \quad 3065(-\mathrm{C}-\mathrm{H}), \quad 785(\mathrm{C}-\mathrm{Cl}) . \quad$ Anal. Calcd. For $\mathrm{C}_{23} \mathrm{H}_{17} \mathrm{ClN}_{2} \mathrm{O}_{3} \mathrm{~S}$ : C, 63.17; H, 3.89; N, 6.40 Found: C, 63.22; H, 3.98; N, 6.35. mp $186^{\circ} \mathrm{C}$, Yield: $60 \%$

1-[4-(3-Benzo[1,3]dioxol-5-yl-2-propenone-1-yl)-'phenyl]-3-(4-chloro-phenyl)- thiourea $(\mathbf{5 j})$

${ }^{1}$ H NMR: 4.9(s, 2H), 5.67(d, 1H), 5.72(d, 1H), 6.14(d, 1H), 6.19(d, 1H), 6.28( s, 1H), 6.41(d, $2 \mathrm{H}), 6.63(\mathrm{~d}, 2 \mathrm{H}), 6.72(\mathrm{~d}, 2 \mathrm{H}), 6.83(\mathrm{~d}, 2 \mathrm{H}), 7.84(\mathrm{~s}, 1 \mathrm{H}), 8.23(\mathrm{~s}, 1 \mathrm{H})$. IR $\left(\mathbf{K B r} \mathbf{~ c m}^{-\mathbf{1}}\right)$ : $1680(-$ $\mathrm{C}=\mathrm{O}), \quad 1502(-\mathrm{C}=\mathrm{C}), 1219$ (-C-O), 1535(C=S), 3406(-NH), 3269(-CS-NH), 2970(-C-H), $3060(-\mathrm{C}-\mathrm{H}), 829(\mathrm{C}-\mathrm{Cl})$. Anal. Calcd. For $\mathrm{C}_{23} \mathrm{H}_{17} \mathrm{ClN}_{2} \mathrm{O}_{3} \mathrm{~S}$ : C, 63.17; H, 3.89; N, 6.40 Found: $\mathrm{C}, 63.10 ; \mathrm{H}, 3.82 ; \mathrm{N}, 6.35 . \mathbf{m p} 185^{\circ} \mathrm{C}$, Yield: $71 \%$

\section{6. General Preparation of 2-[4-(3-Benzo[1,3]dioxol-5-yl-2-propenone)-phenylamino]- $\mathrm{N}$-aryl- acetamide (6a-6j)}

A mixture of $3(2.1 \mathrm{~g}, 0.00783 \mathrm{~mol})$ and 2-chloro-N-arylacetamide $(0.00783 \mathrm{~mol})$ in THF $(10 \mathrm{~mL})$ were refluxed for $4 \mathrm{hrs}$. Progress of reaction was monitored by TLC using ethanol: toluene (1:4) as eluent. After the completion of reaction, the content was added to cold water. The solid product was obtained and filtered, dried and purified by crystallization from Ethanol.

2-[4-(3-Benzo[1,3]dioxol-5-yl-2-propenone)-phenylamino]-N-phenyl-acetamide (6a) ${ }^{1}$ H NMR: 3.73(s, 2H), 5.61(s, 2H), 5.89(d, 1H), 5.91(d, 1H), 6.68(d, 1H), 6.78(d, 1H), 6.79(s, 1H), 6.81(d, 2H), 6.92(d, 2H), 7.14(d, 2H), 7.26(dd, 2H), 7.48(ddd, 1H), 7.73(s, 1H), 7.75(s, 1H). IR (KBr cm $\left.{ }^{-1}\right)$ : 1683(-C=O), 1519(-C=C), 1236(-C-O), 3261(-NH), 3458(-CO$\mathrm{NH}), 2987(-\mathrm{C}-\mathrm{H}), 3078(-\mathrm{C}-\mathrm{H})$. Anal. Calcd. For $\mathrm{C}_{24} \mathrm{H}_{20} \mathrm{~N}_{2} \mathrm{O}_{4}$ : C, 71.92; H, 4.99; N, 6.99 Found: C, 71.87; H, 4.93; N, 6.95. mp $162{ }^{\circ} \mathrm{C}$, Yield: $73 \%$

\section{2-[4-(3-Benzo[1,3]dioxol-5-yl-2-propenone)-phenylamino]-N-(2-fluoro-phenyl)-} acetamide (6b)

${ }^{1}$ H NMR: $3.69(\mathrm{~s}, 2 \mathrm{H}), 4.78(\mathrm{~s}, 2 \mathrm{H}), 5.79(\mathrm{~d}, 1 \mathrm{H}), 5.82(\mathrm{~d}, 1 \mathrm{H}), 6.66(\mathrm{~d}, 1 \mathrm{H}), 6.69(\mathrm{~d}, 1 \mathrm{H})$, 6.81(s, 1H), 6.8(d, 2H), 6.88(d, 2H), 7.24(dd, 1H), 7.31(dd, 1H), 7.33(dd, 1H), 7.46(dd, 1H), 7.74(s, 1H), 7.86(s, 1H). IR (KBr cm $\left.{ }^{-1}\right): 1691(-\mathrm{C}=\mathrm{O}), 1523(-\mathrm{C}=\mathrm{C}), 1241(-\mathrm{C}-\mathrm{O}), 3271(-$ $\mathrm{NH}), 3467(-\mathrm{CO}-\mathrm{NH}), 2997(-\mathrm{C}-\mathrm{H}), 3083(-\mathrm{C}-\mathrm{H}), 715(\mathrm{C}-\mathrm{F})$. Anal. Calcd. For $\mathrm{C}_{24} \mathrm{H}_{19} \mathrm{FN}_{2} \mathrm{O}_{4}$ : C, 68.83; H, 4.54; N, 6.69 Found: C, 68.88; H, 4.49; N, 6.64. mp $150{ }^{\circ} \mathrm{C}$, Yield: $58 \%$

\section{2-[4-(3-Benzo[1,3]dioxol-5-yl-2-propenone)-phenylamino]-N-(3-fluoro-phenyl)-} acetamide (6c)

${ }^{1}$ H NMR: 3.74(s, 2H), 4.81(s, 2H), 5.77(d, 1H), 5.82(d, 1H), 6.68(d, 1H), 6.73(d, 1H), 6.82(s, 1H), 6.78(d, 2H), 6.85(d, 2H), 7.18(m, 1H), 7.31(dd, 1H), 7.41(dd, 1H), 7.44(d, 1H), 7.75(s, 1H), 7.8(s, 1H). IR $\left(\mathbf{K B r} \mathbf{~ c m}^{-1}\right): 1692(-\mathrm{C}=\mathrm{O}), 1527(-\mathrm{C}=\mathrm{C}), 1243(-\mathrm{C}-\mathrm{O}), 3275(-\mathrm{NH})$, 3471(-CO-NH), 3004(-C-H), 3085(-C-H), 744 (C-F). Anal. Calcd. For $\mathrm{C}_{24} \mathrm{H}_{19} \mathrm{FN}_{2} \mathrm{O}_{4}$ : C, 68.83 ; H, 4.54; N, 6.69 Found: C, 68.79; H, 4.50; N, 6.62. mp $147^{\circ} \mathrm{C}$, Yield: $63 \%$

\section{2-[4-(3-Benzo[1,3]dioxol-5-yl-2-propenone)-phenylamino]-N-(4-fluoro-phenyl)- acetamide (6d)}

${ }^{1}$ H NMR: $3.76(\mathrm{~s}, 2 \mathrm{H}), 4.73(\mathrm{~s}, 2 \mathrm{H}), 5.71(\mathrm{~d}, 1 \mathrm{H}), 5.83(\mathrm{~d}, 1 \mathrm{H}), 6.66(\mathrm{~d}, 1 \mathrm{H}), 6.7(\mathrm{~d}, 1 \mathrm{H}), 6.83(\mathrm{~s}$, $1 \mathrm{H}), 6.75(\mathrm{~d}, 2 \mathrm{H}), 6.83(\mathrm{~d}, 2 \mathrm{H}), 7.25(\mathrm{~d}, 2 \mathrm{H}), 7.36(\mathrm{~d}, 2 \mathrm{H}), 7.54(\mathrm{~s}, 1 \mathrm{H}), 7.8(\mathrm{~s}, 1 \mathrm{H}) . \mathbf{I R}\left(\mathbf{K B r} \mathbf{~ c m}^{-}\right.$ $\left.{ }^{1}\right)$ : $1695(-\mathrm{C}=\mathrm{O}), 1531(-\mathrm{C}=\mathrm{C}), 1245(-\mathrm{C}-\mathrm{O}), 3277(-\mathrm{NH}), 3474(-\mathrm{CO}-\mathrm{NH}), 3007(-\mathrm{C}-\mathrm{H}), 3089(-$ 
C-H), 833(C-F). Anal. Calcd. For $\mathrm{C}_{24} \mathrm{H}_{19} \mathrm{FN}_{2} \mathrm{O}_{4}$ : C, 68.83; H, 4.54; N, 6.69 Found: C, 68.90; H, 4.48; N, 6.74. mp $143{ }^{\circ} \mathrm{C}$, Yield: $65 \%$

2-[4-(3-Benzo[1,3]dioxol-5-yl-2-propenone)-phenylamino]-N-o-tolyl-acetamide (6e)

${ }^{1}$ H NMR: $2.32(\mathrm{~s}, 3 \mathrm{H}), 3.68(\mathrm{~s}, 2 \mathrm{H}), 4.76(\mathrm{~s}, 2 \mathrm{H}), 5.69(\mathrm{~d}, 1 \mathrm{H}), 5.8(\mathrm{~d}, 1 \mathrm{H}), 6.67(\mathrm{~d}, 1 \mathrm{H}), 6.71(\mathrm{~d}$, $1 \mathrm{H}), 6.77(\mathrm{~s}, 1 \mathrm{H}), 6.81(\mathrm{~d}, 2 \mathrm{H}), 6.86(\mathrm{~d}, 2 \mathrm{H}), 7.18(\mathrm{dd}, 1 \mathrm{H}), 7.26(\mathrm{dd}, 1 \mathrm{H}), 7.42(\mathrm{dd}, 1 \mathrm{H}), 7.48(\mathrm{dd}$, $1 \mathrm{H}), 7.8(\mathrm{~s}, 1 \mathrm{H}), 8.3(\mathrm{~s}, 1 \mathrm{H})$. IR $\left(\mathbf{K B r ~ c m}^{-1}\right): 1697(-\mathrm{C}=\mathrm{O}), 1533(-\mathrm{C}=\mathrm{C}), 1247(-\mathrm{C}-\mathrm{O}), 3281(-$ $\mathrm{NH}), \quad 3477(-\mathrm{CO}-\mathrm{NH}), \quad 3101(-\mathrm{C}-\mathrm{H}), \quad 3091(-\mathrm{C}-\mathrm{H}), \quad 510\left(\mathrm{Ar}_{-} \mathrm{CH}_{3}\right)$. Anal. Calcd. For $\mathrm{C}_{25} \mathrm{H}_{22} \mathrm{~N}_{2} \mathrm{O}_{4}$ : C, 72.38; H, 5.30; N, 6.75, Found: C, 72.33; H, 5.35; N, 6.70. mp $148{ }^{\circ} \mathrm{C}$, Yield : $78 \%$

\section{2-[4-(3-Benzo[1,3]dioxol-5-yl-2-propenone)-phenylamino]-N-m-tolyl-acetamide (6f)}

${ }^{1}$ H NMR: 2.38(s, 3H), 3.69(s, 2H), 4.77(s, 2H), 5.68(d, 1H), 5.85(d, 1H), 6.65(d, 1H), 6.73(d, 1H), 6.78(s, 1H), 6.84(d, 2H), 6.91(d, 2H), 7.17(s, 1H), 7.31(dd, 1H), 7.39(dd, 1H), 7.46(ddd, 1H), 7.84( s, 1H), 8.26( s, 1H). IR $\left(\mathbf{K B r ~ c m}^{-1}\right)$ : 1701(-C=O), 1534(-C=C), 1249($\mathrm{C}-\mathrm{O}), 3286(-\mathrm{NH}), 3479(-\mathrm{CO}-\mathrm{NH}), 3105(-\mathrm{C}-\mathrm{H}), 3096(-\mathrm{C}-\mathrm{H}), 573\left(\mathrm{Ar}_{-} \mathrm{CH}_{3}\right)$. Anal. Calcd. For $\mathrm{C}_{25} \mathrm{H}_{22} \mathrm{~N}_{2} \mathrm{O}_{4}$ : C, 72.38; H, 5.30; N, 6.75, Found: C, 72.43; H, 5.25; N, 6.80. mp $144^{\circ} \mathrm{C}$, Yield: $72 \%$

2-[4-(3-Benzo[1,3]dioxol-5-yl-2-propenone)-phenylamino]-N-p-tolyl-acetamide (6g)

${ }^{1}$ H NMR: 2.34(s, 3H), 3.71(s, 2H), 4.76(s, 2H), 5.69(d, 1H), 5.79(d, 1H), 6.67(d, 1H), 6.76(d, 1H), 6.8(s, 1H), 6.87(d, 2H), 6.94(d, 2H), 7.24(d, 2H), 7.36(d, 2H), 7.9(s, 1H), 8.31(s, $1 \mathrm{H})$. IR $\left(\mathrm{KBr} \mathrm{cm}^{-1}\right)$ : $1707(-\mathrm{C}=\mathrm{O}), 1537(-\mathrm{C}=\mathrm{C}), 1252(-\mathrm{C}-\mathrm{O}), 3289(-\mathrm{NH}), 3482(-\mathrm{CO}-\mathrm{NH})$, $3107(-\mathrm{C}-\mathrm{H}), 3105(-\mathrm{C}-\mathrm{H}), 823\left(\mathrm{Ar}-\mathrm{CH}_{3}\right)$ Anal. Calcd. For $\mathrm{C}_{25} \mathrm{H}_{22} \mathrm{~N}_{2} \mathrm{O}_{4}$ : C, 72.38; H, 5.30; N, 6.75, Found: C, 72.49; H, 5.38; N, 6.69. mp $145{ }^{\circ} \mathrm{C}$, Yield: $70 \%$

\section{2-[4-(3-Benzo[1,3]dioxol-5-yl-2-propenone)-phenylamino]-N-(2-chloro-phenyl)-} acetamide $(6 \mathrm{~h})$

${ }^{1}$ H NMR: 3.72(s, 2H), 4.81(s, 2H), 5.82(d, 1H), 5.89(d, 1H), 6.64(d, 1H), 6.71(d, 1H), 6.79(s, 1H), 6.79(d, 2H), 6.89(d, 2H), 7.23(dd, 1H), 7.29(dd, 1H), 7.34(ddd, 1H), 7.44(dd, $1 \mathrm{H}), 7.76(\mathrm{~s}, 1 \mathrm{H}), 7.9(\mathrm{~s}, 1 \mathrm{H})$. IR $\left(\mathbf{K B r ~ c m}^{-1}\right)$ : 1685(-C=O), 1512(-C=C), 1239(-C-O), 3265($\mathrm{NH}), 3461(-\mathrm{CO}-\mathrm{NH}), 2991(-\mathrm{C}-\mathrm{H}), 3072(-\mathrm{C}-\mathrm{H}), 729(\mathrm{C}-\mathrm{Cl})$. Anal. Calcd. For $\mathrm{C}_{24} \mathrm{H}_{19} \mathrm{ClN}_{2} \mathrm{O}_{4}$ : C, 66.22; H, 4.36; N, 6.43 Found: C, 66.29; H, 4.40; N, 6.39. mp $160{ }^{\circ} \mathrm{C}$, Yield: $69 \%$

\section{2-[4-(3-Benzo[1,3]dioxol-5-yl-2-propenone)-phenylamino]-N-(3-chloro-phenyl)-} acetamide (6i)

${ }^{1}$ H NMR: 3.79(s, 2H), 4.78(s, 2H), 5.78(d, 1H), 5.84(d, 1H), 6.66(d, 1H), 6.71(d, 1H), 6.81(s, 1H), 6.76(d, 2H), 6.84(d, 2H), 7.18(m, 1H), 7.28(dd, 1H), 7.38(ddd, 1H), 7.43(d, 1H), 7.74(s, 1H), 8.1(s, 1H). IR (KBr cm $\left.{ }^{-1}\right): 1687(-\mathrm{C}=\mathrm{O}), 1515(-\mathrm{C}=\mathrm{C}), 1241(-\mathrm{C}-\mathrm{O}), 3267(-\mathrm{NH})$, 3463(-CO-NH), 2993(-C-H), 3075(-C-H), 782(C-Cl). Anal. Calcd. For $\mathrm{C}_{24} \mathrm{H}_{19} \mathrm{ClN}_{2} \mathrm{O}_{4}$ : C, 66.22; H, 4.36; N, 6.43 Found: C, 66.18; H, 4.30; N, 6.48. mp $166^{\circ} \mathrm{C}$, Yield: $65 \%$

\section{2-[4-(3-Benzo[1,3]dioxol-5-yl-2-propenone)-phenylamino]-N-(4-chloro-phenyl)-} acetamide $(6 \mathbf{j})$

${ }^{1}$ H NMR: 3.81(s, 2H), 4.74(s, 2H), 5.74(d, 1H), 5.81(d, 1H), 6.64(d, 1H), 6.69(d, 1H), 6.82(s, 1H), 6.74(d, 2H), 6.81(d, 2H), 7.24(d, 2H), 7.31(d, 2H), 7.52(s, 1H), 7.79(s, 1H). IR $\left(\mathrm{KBr} \mathbf{~ c m}^{-1}\right)$ : 1689(-C=O), 1521(-C=C), 1238(-C-O), 3269(-NH), 3465(-CO-NH), 2995(-C- 
H), 3081(-C-H), 820(C-Cl). Anal. Calcd. For $\mathrm{C}_{24} \mathrm{H}_{19} \mathrm{ClN}_{2} \mathrm{O}_{4}$ : C, 66.22; H, 4.36; N, 6.43 Found: C, 66.29; H, 4.40; N, 6.50. mp $154{ }^{\circ} \mathrm{C}$, Yield: $68 \%$

\section{ANTIBACTERIAL ACTIVITY}

The minimum inhibitory concentration (MIC) of the synthesized compound were tested against two representative Gram-positive (Staphylococcus aureus MTCC 443 and Staphylococcus pyogenus MTCC 443) and two Gram-negative (Escherichia coli MTCC 442 and Pseudomonas aeruginosa MTCC 443) and assayed in vitro using broth micro dilution method by standards gradual dilution starting from $(250,200,125,100,50,25,12.5,6.25$, $3.125) \mu \mathrm{g} / \mathrm{mL}$.

Ciprofloxacin and Chloramphenicol were used as reference antibacterial agents. Solution of the test compounds and reference drugs were dissolved in DMSO. The in vitro results of the antibacterial activity of the newly synthesized 30 compounds are presented in Table 1.

Some of the compounds displayed moderate the good antibacterial activity in the range of $62.5-125 \mu \mathrm{g} / \mathrm{ml}$. Here the Gram-negative bacteria $E$. coli appered with relative high sensitivity towards 4-fluro, 2-chloro and 4-chloro group substituted analogues $4 \mathrm{~d}, 4 \mathrm{~h}, 4 \mathrm{j}, 5 \mathrm{~d}$, $5 \mathrm{~h}, 5 \mathrm{j}, 6 \mathrm{~d}, 6 \mathrm{~h}$ and $6 \mathrm{j}$ at $100 \mu \mathrm{g} / \mathrm{ml}$ MIC level. With regard to the activity against $P$. aeruginosa, the best inhibition was displayed by compound bearing 2 -fluoro group $4 \mathrm{~b}, 5 \mathrm{~b}$ and $6 \mathrm{~b}(62.5 \mu \mathrm{g} / \mathrm{ml})$.

On the other hand investigation of antibacterial activity of the synthesized compounds against few Gram-positive strains related that $S$. pyogenus is more sensitive against most of the synthesized analogous as compared to $S$. aureus. It was found that present of 2-fluro $(62.5$ $\mu \mathrm{g} / \mathrm{ml})$ and 2-methyl group $(100 \mu \mathrm{g} / \mathrm{ml})$ on para position of $4 \mathrm{~b}, 4 \mathrm{e}, 5 \mathrm{~b}, 5 \mathrm{e}, 6 \mathrm{~b}$ and $6 \mathrm{e}$ accelerate the antibacterial activity against $S$. pyosens. More over the Gram-negative bacteria S.aureus showed relative high sensitivity towards the analogs $4 \mathrm{a}, 4 \mathrm{f}, 5 \mathrm{a}, 5 \mathrm{f}, 6 \mathrm{a}$ and $6 \mathrm{f}$ at 100 $\mu \mathrm{g} / \mathrm{ml}$ MIC level.

Table 1. Antibacterial activity of title compounds.

\begin{tabular}{|c|c|c|c|c|c|}
\hline \multirow{3}{*}{$\begin{array}{l}\text { Compd. } \\
\text { No }\end{array}$} & \multicolumn{5}{|c|}{ ANTIBACTERIAL ACTIVITY TABLE } \\
\hline & \multicolumn{5}{|c|}{ MINIMUM INHIBITORY CONCENTRATION } \\
\hline & $-\mathrm{R}$ & $\begin{array}{c}\text { E. coli } \\
\text { MTCC } 442 \\
\mu \mathrm{g} / \mathrm{ml}\end{array}$ & $\begin{array}{c}P . \text { aeruginosa } \\
\text { MTCC } 443 \\
\mu \mathrm{g} / \mathrm{ml}\end{array}$ & $\begin{array}{c}\text { S. aureus } \\
\text { MTCC } 443 \\
\mu \mathrm{g} / \mathrm{ml}\end{array}$ & $\begin{array}{c}\text { S. pyogenus } \\
\text { MTCC } 443 \\
\mu \mathrm{g} / \mathrm{ml}\end{array}$ \\
\hline $4 a$ & $-\mathrm{H}$ & 250 & 250 & 100 & 100 \\
\hline $4 \mathrm{~b}$ & $2-\mathrm{F}$ & 100 & 62.5 & 250 & 62.5 \\
\hline $4 \mathrm{c}$ & $3-\mathrm{F}$ & 200 & 200 & 200 & 200 \\
\hline $4 d$ & $4-\mathrm{F}$ & 100 & 100 & 250 & 125 \\
\hline $4 \mathrm{e}$ & $2-\mathrm{CH}_{3}$ & 250 & 200 & 125 & 100 \\
\hline
\end{tabular}




\begin{tabular}{|c|c|c|c|c|c|}
\hline $4 \mathrm{f}$ & $3-\mathrm{CH}_{3}$ & 200 & 125 & 100 & 250 \\
\hline $4 \mathrm{~g}$ & $4-\mathrm{CH}_{3}$ & 125 & 100 & 62.5 & 125 \\
\hline $4 \mathrm{~h}$ & $2-\mathrm{Cl}$ & 100 & 200 & 200 & 200 \\
\hline $4 \mathrm{i}$ & $3-\mathrm{Cl}$ & 200 & 250 & 125 & 200 \\
\hline $4 j$ & $4-\mathrm{Cl}$ & 100 & 250 & 200 & 125 \\
\hline $5 a$ & $-\mathrm{H}$ & 100 & 250 & 100 & 125 \\
\hline $5 b$ & $2-\mathrm{F}$ & 250 & 62.5 & 250 & 62.5 \\
\hline $5 c$ & $3-\mathrm{F}$ & 100 & 100 & 200 & 250 \\
\hline $5 d$ & $4-F$ & 100 & 250 & 250 & 62.5 \\
\hline $5 e$ & $2-\mathrm{CH}_{3}$ & 250 & 200 & 125 & 100 \\
\hline $5 f$ & $3-\mathrm{CH}_{3}$ & 200 & 125 & 100 & 250 \\
\hline $5 \mathrm{~g}$ & $4-\mathrm{CH}_{3}$ & 62.5 & 100 & 250 & 250 \\
\hline $5 \mathrm{~h}$ & $2-\mathrm{Cl}$ & 100 & 200 & 200 & 200 \\
\hline $5 \mathrm{i}$ & $3-\mathrm{Cl}$ & 200 & 250 & 125 & 200 \\
\hline $5 \mathrm{j}$ & $4-\mathrm{Cl}$ & 100 & 250 & 100 & 125 \\
\hline $6 a$ & $-\mathrm{H}$ & 125 & 250 & 100 & 125 \\
\hline $6 b$ & $2-\mathrm{F}$ & 250 & 62.5 & 250 & 62.5 \\
\hline $6 c$ & $3-\mathrm{F}$ & 100 & 500 & 200 & 200 \\
\hline $6 \mathrm{~d}$ & $4-F$ & 100 & 250 & 250 & 125 \\
\hline $6 e$ & $2-\mathrm{CH}_{3}$ & 250 & 200 & 125 & 100 \\
\hline $6 f$ & $3-\mathrm{CH}_{3}$ & 100 & 125 & 100 & 250 \\
\hline $6 \mathrm{~g}$ & $4-\mathrm{CH}_{3}$ & 125 & 100 & 100 & 250 \\
\hline $6 \mathrm{~h}$ & $2-\mathrm{Cl}$ & 100 & 125 & 200 & 200 \\
\hline $6 \mathrm{i}$ & $3-\mathrm{Cl}$ & 200 & 250 & 125 & 200 \\
\hline $6 \mathrm{j}$ & $4-\mathrm{Cl}$ & 100 & 125 & 200 & 125 \\
\hline \multirow[t]{2}{*}{$\begin{array}{c}\text { Standard } \\
\text { Drug } \\
\end{array}$} & Ciprofloxacin & 25 & 25 & 50 & 50 \\
\hline & $\begin{array}{c}\text { Chloramphen } \\
\text { icol }\end{array}$ & 50 & 50 & 50 & 50 \\
\hline
\end{tabular}




\section{ANTIFUNGAL ACTIVITY}

The minimum inhibitory concentration (MIC) of the synthesized compound was tested against fungi (Candida albicans MTCC 227, Aspergillus niger MTCC 282 and Aspergillus clavatus MTCC 1323) and assayed in vitro using broth micro dilution method standards with gradual dilution starting from $(250,200,125,100,50,25,12.5,6.25,3.125) \mu \mathrm{g} / \mathrm{mL}$. Nystatin and Greseofulvin were used as reference antibacterial agents. Solution of the test compounds and reference drugs were dissolved in DMSO. The in vitro results of the antifungal activity of the newly synthesized 30 compounds are presented in Table 2.

Some of the compounds displayed moderate the good antibacterial activity. The in vitro antifungle activity of the synthesized analogues indicated that halogen and methyl group substituted analogs 4d, 4e, 4i, 5d, 5e, 5i, 6d, 6e and 6i have shown high efficacy against $C$. albicans \& A. clavatus at $100 \mathrm{ug} / \mathrm{ml}$ MIC level.

Table 2. Antifungal activity of title compounds.

\begin{tabular}{|c|c|c|c|c|}
\hline \multirow{3}{*}{$\begin{array}{c}\text { Compd. } \\
\text { No. }\end{array}$} & \multicolumn{4}{|c|}{ ANTIFUNGAL ACTIVITY TABLE } \\
\hline & \multicolumn{4}{|c|}{ MINIMUM INHIBITORY CONCENTRATION } \\
\hline & $-\mathrm{R}$ & $\begin{array}{c}\text { C. albicans } \\
\text { MTCC } 227 \\
\mu \mathrm{g} / \mathrm{ml}\end{array}$ & $\begin{array}{c}\text { A. niger } \\
\text { MTCC } 282 \\
\mu \mathrm{g} / \mathrm{ml}\end{array}$ & $\begin{array}{c}\text { A. clavatus } \\
\text { MTCC } 1323 \\
\mu \mathrm{g} / \mathrm{ml}\end{array}$ \\
\hline $4 \mathrm{a}$ & $-\mathrm{H}$ & 500 & $>1000$ & 1000 \\
\hline $4 \mathrm{~b}$ & $2-\mathrm{F}$ & 500 & 500 & $>1000$ \\
\hline $4 \mathrm{c}$ & $3-\mathrm{F}$ & 1000 & 500 & $>1000$ \\
\hline $4 \mathrm{dd}$ & 4-F & 100 & 250 & 100 \\
\hline $4 \mathrm{e}$ & $2-\mathrm{CH}_{3}$ & 100 & 1000 & 100 \\
\hline $4 \mathrm{f}$ & $3-\mathrm{CH}_{3}$ & 500 & $>1000$ & 500 \\
\hline $4 \mathrm{~g}$ & $4-\mathrm{CH}_{3}$ & 250 & 500 & 500 \\
\hline $4 \mathrm{~h}$ & $2-\mathrm{Cl}$ & 500 & $>1000$ & 1000 \\
\hline $4 \mathrm{i}$ & $3-\mathrm{Cl}$ & 100 & 500 & 100 \\
\hline $4 \mathrm{j}$ & 4-Cl & 250 & 1000 & $>1000$ \\
\hline$\overline{5 a}$ & $-\mathrm{H}$ & 250 & 2500 & 250 \\
\hline $5 b$ & $2-\mathrm{F}$ & 200 & 250 & 500 \\
\hline $5 \mathrm{c}$ & $3-\mathrm{F}$ & 250 & 500 & $>1000$ \\
\hline $5 \mathrm{~d}$ & 4-F & 100 & 250 & 100 \\
\hline $5 \mathrm{e}$ & $2-\mathrm{CH}_{3}$ & 100 & 1000 & 100 \\
\hline $5 \mathrm{f}$ & $3-\mathrm{CH}_{3}$ & 1000 & 200 & 500 \\
\hline $5 \mathrm{~g}$ & $4-\mathrm{CH}_{3}$ & 250 & 500 & 100 \\
\hline $5 \mathrm{hh}$ & $2-\mathrm{Cl}$ & 2500 & 250 & 1000 \\
\hline
\end{tabular}




\begin{tabular}{|c|c|c|c|c|}
\hline $5 \mathrm{i}$ & $3-\mathrm{Cl}$ & 100 & 500 & 100 \\
\hline $5 \mathrm{j}$ & $4-\mathrm{Cl}$ & 250 & 500 & $>1000$ \\
\hline $6 a$ & $-\mathrm{H}$ & 125 & 1000 & 250 \\
\hline $6 \mathrm{~b}$ & $2-\mathrm{F}$ & 100 & 200 & $>1000$ \\
\hline $6 c$ & $3-\mathrm{F}$ & 250 & 500 & 100 \\
\hline $6 \mathrm{~d}$ & $4-\mathrm{F}$ & 100 & 250 & 100 \\
\hline $6 e$ & $2-\mathrm{CH}_{3}$ & 100 & 1000 & 100 \\
\hline $6 f$ & $3-\mathrm{CH}_{3}$ & 250 & 200 & 500 \\
\hline $6 g$ & $4-\mathrm{CH}_{3}$ & 100 & 500 & 250 \\
\hline $6 \mathrm{~h}$ & $2-\mathrm{Cl}$ & 62.5 & 250 & 1000 \\
\hline $6 \mathrm{i}$ & $3-\mathrm{Cl}$ & 100 & 500 & 100 \\
\hline $6 \mathrm{j}$ & $4-\mathrm{Cl}$ & 125 & 250 & $>1000$ \\
\hline \multirow[t]{2}{*}{$\begin{array}{c}\text { Standard } \\
\text { Drug }\end{array}$} & Nystatin & 100 & 100 & 100 \\
\hline & Greseofulvin & 500 & 100 & 100 \\
\hline
\end{tabular}

\section{CONCLUSIONS}

In conclusion, a new class of chalcone based urea, thiourea, acetamidd derivatives were synthesized and evaluated as antibacterial agents. The newly synthesized compounds exhibited promising antibacterial activities against two representative Gram-positive (Staphylococcus aureus and Staphylococcus pyogenus) and two Gram-negative (Escherichia coli and Pseudomonas aeruginosa). These results makes novel chalcone and their urea, thiourea and acetamided derivatives interesting lead molecules for further synthetic and biological evaluation. It can be concluded that this class of compounds certainly holds great promise towards the pursuit to discover novel classes of antifungal agents. Further studies to acquire more information concerning structure - activity relationships are in progress.

\section{Acknowledgements}

The authors are thankful to Dr (Ms.) N. K. Shah, Head of the Chemistry Department and Director of School of Sciences, Gujarat University for all the support and facilities. The authors also express their sincere thanks to the Oxygen health care for spectral analysis. Dhruvin Shah and Nirali Mewada are also thankful to UGC for providing financial assistance.

\section{References}

[1] J.H. Cheng, C.F. Hung, S.C. Yang, J.P. Wang, S.J. Won, C.N. Lin, Bioorg. Med. Chem. 16 (2008) 7270-7276.

[2] K. H. Chikhalia, M. J. Patel, D. B. Vashi, ARKIVOC 13 (2008) 189-197.

[3] C. Zhan, J. Yang, Pharmacol. Res. 53 (2006) 303-309. 
[4] M. Liu, P. Wilairat, M.L. Go, J. Med. Chem. 44 (2001) 4443-4452.

[5] M.L. Go, X. Wu, X.L. Liu, Curr. Med. Chem. 12 (2001) 481-499.

[6] V.S. Koneni, K. Abdhesh, K. Manoj, S. Jayanta, S. Sudhir, Bioorg. Med. Chem. Lett. 20 (2010) 7205-7211.

[7] P. A. Yonova, G. M. Stoilkova, J. Plant Growth Regul. 23 (2004) 280-291.

[8] Y. J. Kim, J. H. Ryu, Y. J.Cheon, H. J. Lim, R. Jeon, Bioorg. Med. Chem. Lett. 17 (2007) 3317-3321.

[9] O. Adeoye, A. A. Ayandele, O. A. Odunola, J. Agric. Biol. Sci. 2 (2007) 4-5.

[10] T. K. Venkatachalam, C. Mão, F. M. Uckun, Bioorg. Med. Chem. 12 (2004) 4275- 4284.

[11] J. D. Bloom, R. G. Dushin, K. J. Curran, F. Donahue, E. B. Norton, E. Terefenko, T. R. Jonas, A. A. Ross, B. Feld, S. A. Lang, M. J. Di-Grandi, Bioorg. Med. Chem. 14 (2004) 3401-3406.

[12] C. Furman, J. Lebeau, J. C. Fruchart, J. L. Bernier, P. Duriez, N. Cotelle, E. Teissier, E. J. Biochem. Mol. Toxicol. 15 (2001) 270-278.

[13] J. Rojas, M. Paya, J. N. Domínguez, L. Ferrandiz, Bioorg. Med. Chem. Lett. 12 (2002) 1951-1954.

[14] J. Rojas, M. Paya, L. Devesa, J. N. Domínguez, L. Ferrandiz, Naunyn-Schmiedeberg's Arch. Pharmacol. 368 (2003) 225-233.

[15] A. Araico, M. C. Terencio, M. J. Alcaraz, J. N. Dominguez, C. León, M. L. Ferrándiz, Life Sci. 80 (2007) 2108-2117.

[16] J. N. Dominguéz, C. León, J. Rodrigues, N. Gamboa de Dominguez, J. Gut, P. G. Rosenthal, J. Med. Chem. 48 (2005) 3654-3658.

[17] J.H. Clark, H. L. Holland, J.M. Miller, Tetrahedron Lett. 38 (1978) 3361-3364.

[18] H. S. Bodiwala, S. Sabde, P. Gupta, R. Mukherjee, R. Kumar, P. Garg, K. K. Bhutani, D. Mitram I. P. Singh, Bioorg. Med. Chem. 19 (2011) 1256.

[19] G. Thirunarayanan, K. G. Sekar, International Letters of Chemistry, Physics and Astronomy 10(1) (2013) 18-34.

[20] S. Vijayakumar, R. Arulkumaran, R. Sundararajan, S. P. Sakthinathan, R. Suresh, D. Kamalakkannan, K. Ranganathan, K. Sathiyamoorthy, V. Mala, G. Vanangamudi, G. Thirunarayanan, International Letters of Chemistry, Physics and Astronomy 9(1) (2013) 68-86.

[21] R. Sundararajan, R. Arulkumaran, S. Vijayakumar, D. Kamalakkannan, R. Suresh, S. John Joseph, K. Ranganathan, S. P. Sakthinathan, G. Vanangamudi, G. Thirunarayanan, International Letters of Chemistry, Physics and Astronomy 1 (2014) 67-73.

[22] K. G. Sekar, G. Thirunarayanan, International Letters of Chemistry, Physics and Astronomy 8(3) (2013) 249-258.

[23] G. Thirunarayanan, K. Ravi, International Letters of Chemistry, Physics and Astronomy 14 (2013) 44-57. 
[24] S. P. Sakthinathan, R. Suresh, V. Mala, K.Sathiyamoorthi, D. Kamalakkannan, K. Ranganathan, R. Arulkumaran, S. Vijayakumar, R. Sundararajan, G. Vanangamudi, G. Thirunarayanan, International Letters of Chemistry, Physics and Astronomy 6 (2013) $77-90$.

[25] G. Thirunarayanan, R. Sundararajan, R. Arulkumaran, International Letters of Chemistry, Physics and Astronomy 4 (2014) 82-97. 